\title{
A Research on Mechanisms and Countermeasures of the Food Safety Incidents Occurring on Food Supply Chain
}

\author{
Yongsheng Liu, Qingnan Zhang, Qingqing Li \\ Business School, Beijing Wuzi University, Beijing, China \\ Email: bjwylys@sina.com
}

Received 29 June 2014; revised 22 July 2014; accepted 14 August 2014

Copyright $@ 2014$ by authors and Scientific Research Publishing Inc.

This work is licensed under the Creative Commons Attribution International License (CC BY). http://creativecommons.org/licenses/by/4.0/

(c) (i) Open Access

\begin{abstract}
In recent years, food safety problems have become increasingly serious in China, and major food safety accidents have seriously affected people's daily lives, having negative influences on the healthy development of the food industry and the whole economy. Therefore, it is necessary to analyze existing problems of food safety, further study the main cause of frequent incidents of food safety, and propose the corresponding countermeasures. In this paper, food safety incidents occurring from 2009 to 2013 are taken as the research objects, and the food supply chain is divided into five links. Then comprehensive analyses of food category are made, the main responsibility and the possible causes for food safety accidents occurring on each food supply chain links, aiming at analyzing the accident mechanism and proposing improvement strategies.
\end{abstract}

\section{Keywords}

Food Supply Chain, Food Safety, Mechanism

\section{Introduction}

Food safety, which is directly related to the sound development of the people's health and the social economy, is one of the world's hot issues nowadays. In recent years, Chinese food safety incidents have been occurring frequently, most of which have caused very serious consequences and adverse effects. With the rapid development of Chinese economy, profits and efficiency are becoming a major goal of the food enterprise development, which has brought a lot of food safety hazards. Food from the farm to the consumers, usually go through multiple supply chain links such as plant breeding, circulation, processing, consumption, recycling, etc. Referring to the statistical data, each links have suffered safe problems in different degrees. In this paper, 1054 food safety 
incidents occurring from 2009 to 2013 are taken as the research objects, while, based on the supply chain perspective, we analyze the mechanism of food safety incidents from areas of food category, the main responsibility and the possible causes, in order to propose some improvement strategies in every links.

\section{Literature Review}

Despite that people have concerned about food safety issues extensively, researches from the food supply chain perspective for food safety incidents are rare, the existing literature is mainly focused on the causes, solving strategies, influencing factors and other areas of food risk occurring on food supply chain. As Gu et al. (2005) [1] considered that food safety involves three main market subjects who are regulators (government departments taking part in food safety supervising), the regulated party (including raw material suppliers, manufacturers, transport companies, wholesalers and retailers and other food supply chain links) as well as consumers, and discussed the food safety strategies from the macroscopic level, and the game between regulators and the regulated firms from the medium level. Miao (2013) [2] analyzed food safety regulatory issues occurring in circulation link, and proposed countermeasures that could strengthen the supervision of the food safety in circulation link, based on the particularity of food production and distribution operators in current China. Sun et al. (2012) [3] proposed and demonstrated the concept and meaning, goals and signs, levels and contents as well as difficulties and obstacles of coordinated behaviors for quality safety in breeding as well as slaughter and processing link of high quality pork supply chain. Hastein et al. (2006) [4] analyzed the associated risk factors and causes that may result in risks in farming and production processes after consumers buying fish and fish products, eventually finding that the greatest risk to human health is due to the consumption of raw materials or insufficiently processed fish and fish products, and the risks in the process of fish farming, capturing, producing and processing were discussed.

There is also some literature on the issues of food safety risk from the perspective of the whole food supply chain. Zhang et al. (2013) [5] made a research on the causes and influencing factors of risk that occurred in all links of food quality and safety such as food supply of raw materials, production, processing, packaging, storage, transportation and distribution and sale, and put forward the academic suggestion, but it's lack of empirical validation. While, using empirical research methods, Li (2012) [6], Zhang et al. (2013) [7] and Liu et al. (2011) [8] took a large number of food safety incidents that had actually occurred as samples, and analyzed food supply chain risk factors existing in every links. Using risk matrix analysis method, the latter two conducted a comprehensive assessment of the risk level of risk factors. Similarly, using empirical research methods, Luo et al. (2013) [9] made qualitative and descriptive statistics analyses on 3484 food safety incidents during 2001 to 2011 from food category, supply chain links where risk occurred, essential reason that risk formed, and risk responsibility four main aspects. And the conclusion that the key sources of risk in China are meat, deep-processing sectors, improper amount of elements used, individual producers and operators was made.

Overall, compared to studies in other countries, although Chinese scholars started researching the safety issues of food supply chain were later, in recent years the society and scholars have been paying more and more attention to it, and researches has become increasingly diverse. In terms of the research methods, scholars always used qualitative and theoretical analysis methods to make researches that aimed at the food risk in the particular links of food supply chain. Empirical analysis method was usually used when the whole food supply chain was taken as a study object. But literature of comprehensive analysis about food safety incidents from the perspective of the entire network of the food supply chain is rare, this paper attempts to analyze and explore the issue using empirical methods.

\section{Mechanism of Chinese Food Safety Incidents}

\subsection{Data Sources and Processing}

In this paper, considering internet news related to food safety between January 1, 2009 to December 31, 2013 as objects, we analyze the characteristics of the food safety risk. To ensure objectivity and comprehensiveness of samples, we refer to the information in National Food Quality Safety net as the main source of data and other local food sites as auxiliary information query sites. Then we eliminate duplicate and invalid samples, obtained 1054 valid samples of food safety incidents. The food category, occurring links, regions and causes are random, indicating that the selected samples are scientific, which could reflect the characteristics of the food safety risks 
in China in these 5 years. The reason that the three aspects of links of food safety incidents, the main responsibility and the possible causes whose sum of percent are greater than $100 \%$ is that some of the incidents are caused by several factors.

\subsection{Univariate Analysis to Identify the Mechanism of Food Safety Incidents}

The food supply chain includes supply (production) link, circulation, processing link, consumer link and recycling link, any risks in which will have a negative impact on the entire food supply chain, and hazard food quality and safety. First, we analyze the factors that have an impact on food safety such as supply chain links, food categories, enterprise scales and the possible causes of food safety incidents, which contributes to a more clear understanding of the mechanism of food safety incidents.

\subsubsection{The Supply Chain Links of Food Safety Incidents Occurring}

According to the characteristics of the food supply chain, we classify the food supply chain into five primary links, under which each level is divided into several second links (in Table 1). Because the food safety incidents that result from supervision, inspection and quarantine always occur in circulation links, we classify the 2nd link of food supervision into 1st link of circulation link. The classification of food safety incidents according to food supply chain links is shown in Table 1.

Numbers and frequency that are counted by 2nd links of the food supply chain is shown in Figure 1.

\subsubsection{Food Category Involved in Food Safety Incidents}

We mainly classify food on the basis of food categories in "Hygienic Standards for Uses of Food Additives" (GB2760-2011) and "food quality and safety market access system" (QS). Integrating two standards of food safety and food categories actually involved in the events, we eventually classify food into 26 types. The classification results are shown in Figure 2. As can be seen from Figure 2, meat and meat products (173 times, $16.41 \%$ ) is the most frequent food safety incident, cumulative incidence of 173 times. While, edible oil, oil and its products (75 times, $7.12 \%$ ) as well as beverage category (74 times, $7.02 \%$ ) followed.

\subsubsection{Enterprises Scale Involved in Food Safety Incidents}

To clear the primary responsibility of food safety incidents, according to the statistical characteristics of food safety incidents samples, we divided related subjects into eight types, namely unlicensed operators, individual businesses (certified), small enterprises, large and medium-sized enterprises, home, online store, most enterprise (three or more than three scale enterprises) and unsure. And the statistical proportion of food safety incidents is

\section{Table 1. Occurrence of food safety incidents dividing by food supply chain links.}

\begin{tabular}{|c|c|c|c|}
\hline The first level of food supply chain & The second level of food supply chain & The number of events & Proportion \\
\hline Supply (production) link & Planting/breeding & 45 & $4.12 \%$ \\
\hline Circulation link & $\begin{array}{c}\text { Transportation/storage } \\
\text { Food supervision }\end{array}$ & 27 & $2.47 \%$ \\
\hline Processing link & $\begin{array}{c}\text { Primary processing of food } \\
\text { Comprehensive processing of food }\end{array}$ & 720 & $65.93 \%$ \\
\hline \multirow[b]{2}{*}{ Consumption link } & $\begin{array}{c}\text { Wholesale (sales link) } \\
\text { Retail (sales link) }\end{array}$ & & \\
\hline & $\begin{array}{c}\text { Canteen (catering link) } \\
\text { Catering Industry (catering link) } \\
\text { Home (catering link) }\end{array}$ & 259 & $23.70 \%$ \\
\hline Recycling link & Food recycling & 41 & $3.75 \%$ \\
\hline
\end{tabular}




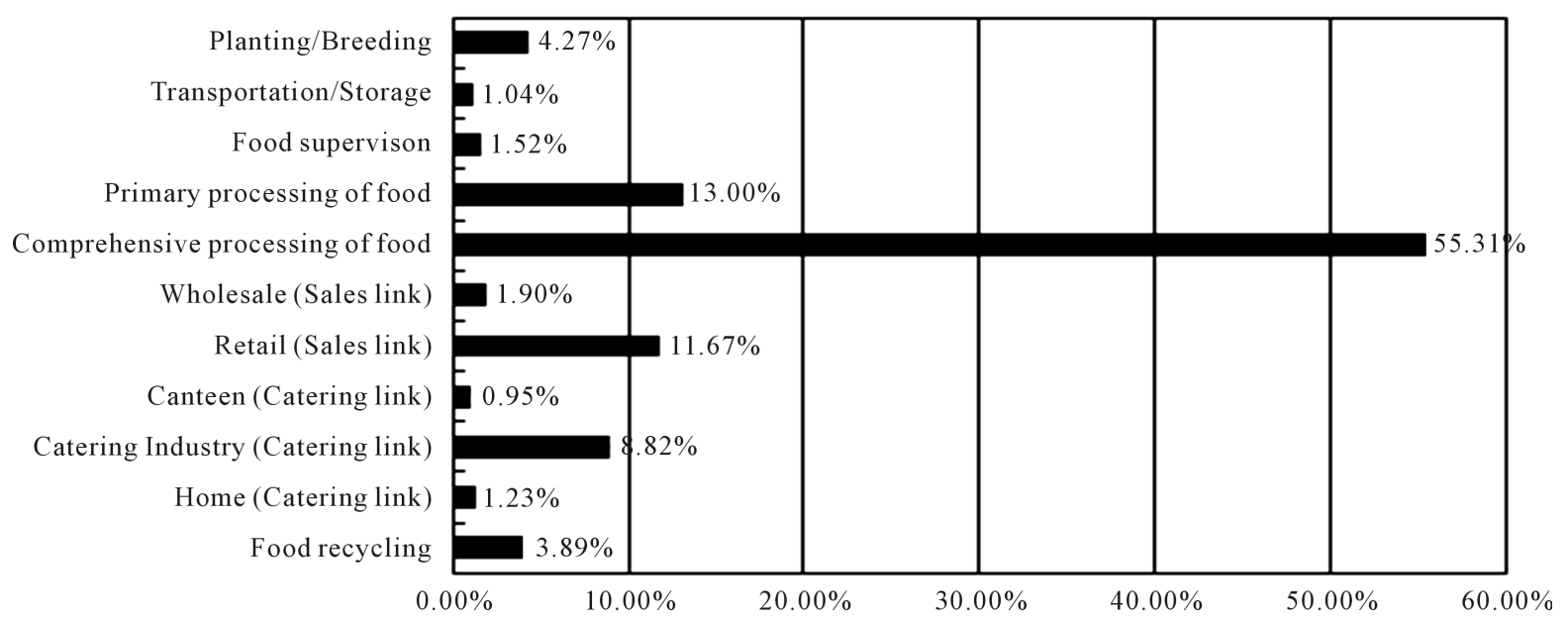

Figure 1. Occurring proportion of food safety incidents in 2nd food supply chain links.

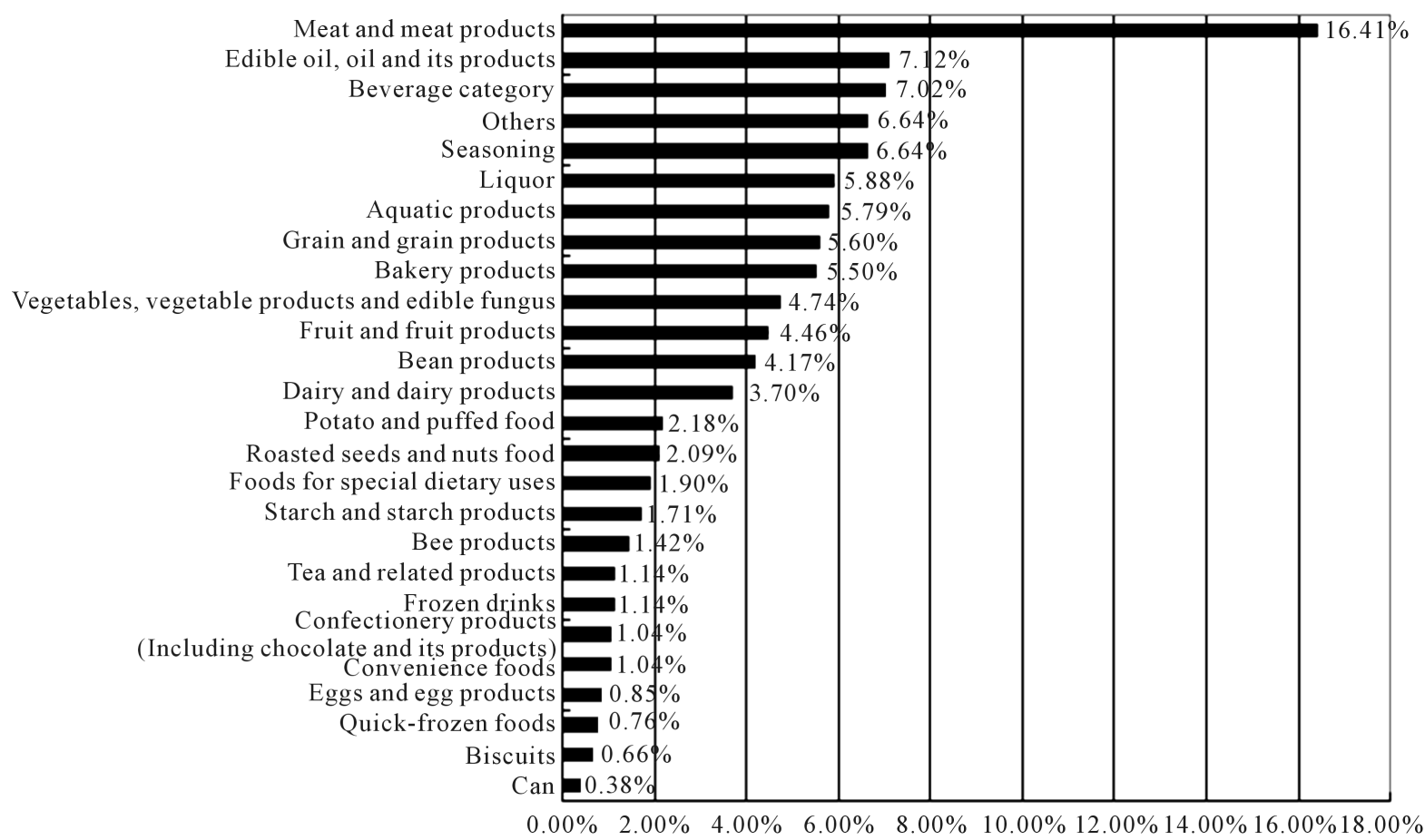

Figure 2. Occurring proportion of food safety incidents by food category.

shown in Figure 3. The top three were large and medium-sized enterprises (268 times, 25.43\%), unlicensed operators (261 times, 24.76\%) and small businesses (170 times, 16.13\%).

\subsubsection{Possible Causes of Food Safety Incidents}

By analyzing the causes of all samples, we conclude 24 kinds of occurring reasons. Then we calculate the proportion of factors occurring, and the proportion of the relevant factors is shown in Figure 4. The top three were excessive and illegal use of other hazards and elements (228 times, 21.63\%), excessive and illegal use of food additives (201 times, 19.07\%), cheat (173 times, 16.45\%).

\subsection{Multi-Factor Analysis to Identify the Mechanism of Food Safety Incidents}

In terms of food supply chain links, the most frequent link where food safety incidents occurred is processing 


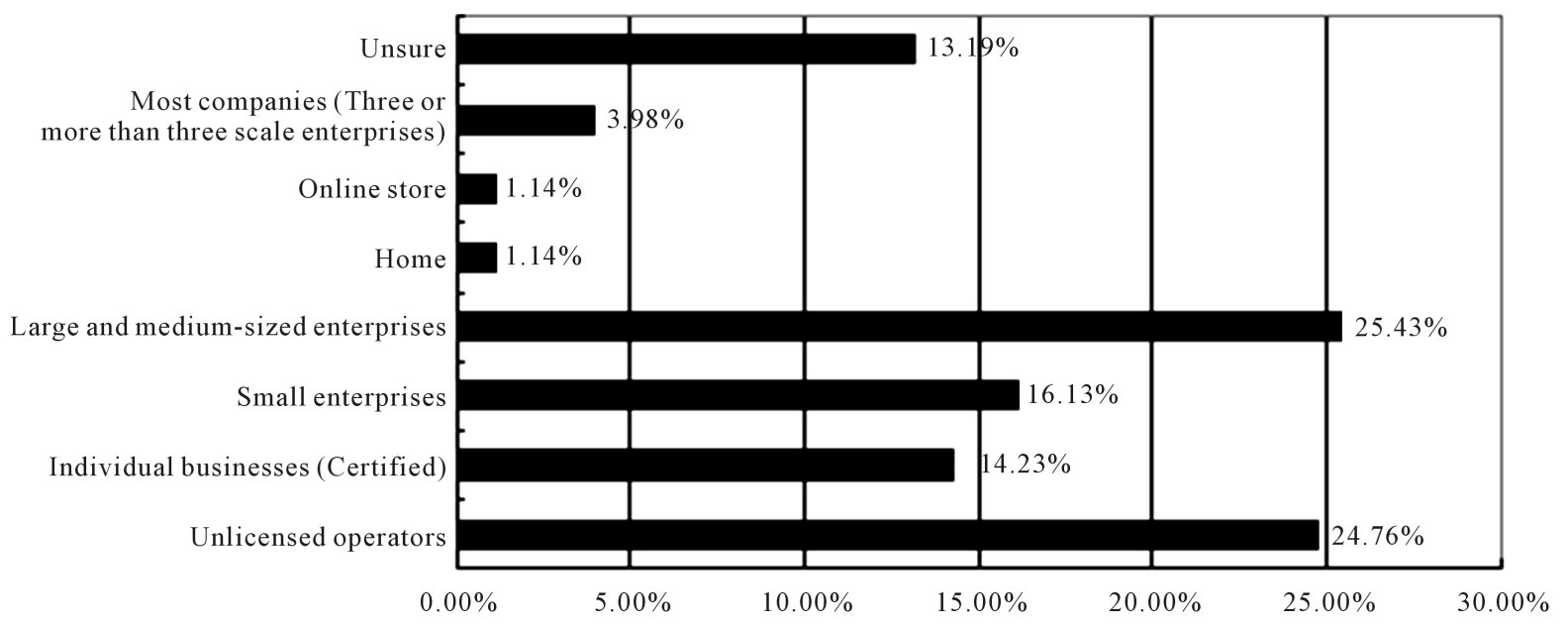

Figure 3. Occurring proportion of food safety incidents by related subjects.

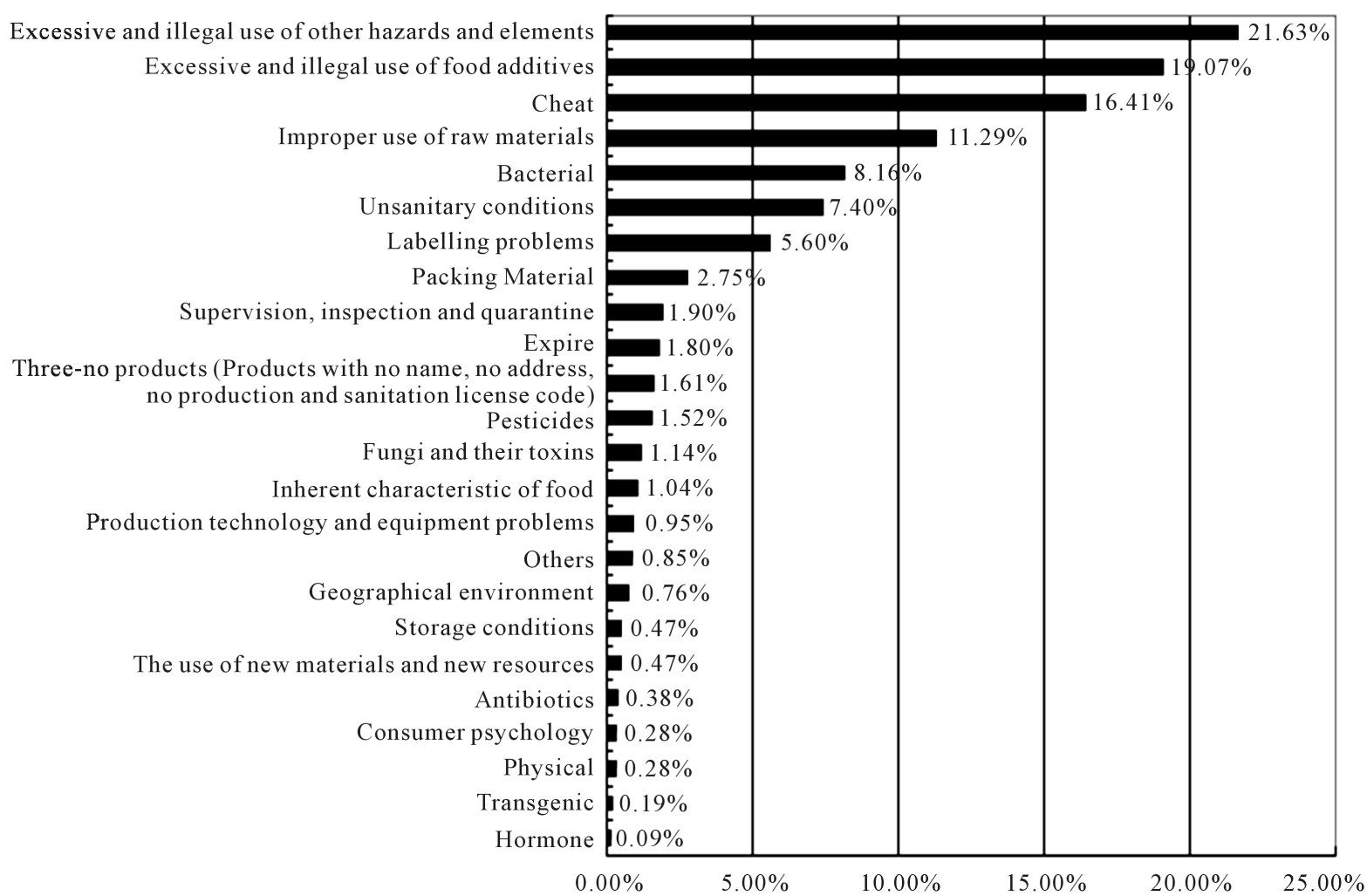

Figure 4. Occurring proportion of food safety incidents causing factors.

link, cumulative incidence of 720 times and accounting for 65.93\%. Then consumption link (259 times, $23.70 \%$ ), supply (production) link (45 times, 4.12\%), recycling link (41 times, 3.75\%) and circulation link (27 times, $2.47 \%$ ) follow by it.

\subsubsection{Food Supply (Production) Link}

Food safety incidents occurring in food supply (production) link are relatively few. There are two reasons accounting for it. On the one hand, it's the natural environment, such as excessive metals and other chemical elements caused by the air pollution or land contamination, which have negative impact on fruits, vegetables and animal husbandry growth. On the other hand, human intentionally add excessive pesticide to fruits and vegeta- 
bles, and feed animals with illegal food additives and other harmful elements such as lean meat powder, antibiotics and other substances that cause hazardous events. Combined with the other three factors, the high frequent food is the meat and meat products, cumulative incidence of 17 times, and a major factor in the occurrence of the problem is excessive and illegal use of food additives as well as excessive and illegal use of other hazards and elements. Then food following by it is vegetables and fruits, mainly due to pesticide residues.

\subsubsection{Food Circulation Link}

Food circulation is the link that occurs the least issues of the entire food supply chain. From the statistical data, the food category in this part has no significant features. But in terms of main responsibility for the incidents, the large and medium-sized enterprises are relatively frequent, which are mainly caused by the transporting temperature, packaging and extrusion and other technical issues.

\subsubsection{Food Processing Link}

Food processing, especially food comprehensive processing, is the disaster area where food safety incidents occur. In the link of food primary processing, edible oil, oil and its products cumulatively occurred of 40 times, and the main responsibility is unlicensed operators, which cumulatively occurred of 40 times, and the relevant factors are mainly illegal use of other hazards and elements, cumulative incidence 50 times. Most events are "black" workshop produced and processed unlicensed waste oil, such as event "In Hunan Yiyang, a 'black' workshop refined 'black lard' that passed quality inspection, mainly flowing to school." Food comprehensive processing is that occurred food safety incidents most frequent, cumulative incidence of 583 and accounting for $55.31 \%$. High-frequency foods are meat and meat products (85 times, $14.58 \%$ ), beverage category (56 times, 9.61\%), seasoning (53 times, 9.09\%), liquor (50 times, 8.58\%), and bakery products (50 times $8.58 \%$ ). The main responsibility that occurred events most frequently is followed by the unlicensed operators, large and medium-sized as well as small enterprises. The main factors leading to incidents are excessive and illegal use of food additives as well as excessive and illegal use of other hazards and elements, both of which cumulatively occurred of 122 times. Then the following is cheat, cumulative incidence of 85 times. As the food comprehensive processing not only attaches higher requirements to operating and skill of personnel, equipment levels of factory and the company's management system and so on, but also is the key for enterprises to create profits, thus causing that some traders do a series of violations to make profits. Most of unlicensed operators, whose sites are usually hidden, do many irregularities such as producing in dirty and messy sanitary conditions, adding hazards illegally, using inferior materials, cheating, etc., know little about food safety and exist a variety of serious problems. Once foods they produce are put into the market, it must lead consumers to serious health hazards. Large and medium-sized enterprises also made many food safety incidents in food comprehensive processing link. Generally, every food types are involved, while, foods that caused the greatest impact were mainly meat and meat products, dairy and dairy products and beverage category. For instance, "CCTV exposed Jiyuan Shuanghui Food Co., Ltd. acquired lean pork incident in 2010”, "a number of dairy companies including Shanghai Panda Dairy Company were exposed excessive melamine incidents again in 2010", "three drinks of Uni-president China Holdings Ltd. were detected plasticizer in 2011”, etc.

\subsubsection{Food Consumption Link}

In food consumption link, the highest incidence event was retail (123 times, 11.26\%) and catering industries (93 times, 8.52\%) links. In retail, meat and meat products are still the most frequent types of food. Main responsibility is large and medium-sized enterprises, total 31 times. The occurring cause is mostly cheating, total 38 times. Integrated several factors, the retailing problems of large and medium-sized enterprises are mostly happened in large supermarket, involving a variety of reasons, and the common are inconsistent with the actual label, the expired products not off the shelf and so on. The main responsibility for the cheating is individual businesses. Because they are small, complex, and fragmented, which makes regulatory difficult, and businesses have low self-discipline and social responsibility, causing purchase channels and product storage conditions are difficult to control, which leads individual businesses to sell fakes.

In the catering industry, the phenomena of individual businesses illegally add "a drop of fragrant", "toner" and other food additives as well as substandard sanitary conditions are common and severe. In addition, large and medium-sized enterprises occur food safety incidents frequent, mainly for unsanitary conditions, such as Kentucky Fried Chicken, Pizza Hut, Yoshinoya, Quanjude and so on. 


\subsubsection{Food Recycling Link}

The problems of food recycling focused on two aspects, one is the recycling of the problem meat (sickness, death, deterioration, decay, etc.) issues; second is the recycling of waste oil, animal offal processing oil (oil produced by simple processing and refining of inferior, outdated and corrupt animal skins, meat, offal). Currently the main responsibility for the occurrence of these two problems is individual businesses and unlicensed operators. Large and medium-sized enterprises occurred only once in these 5 years, that is Henan Huaying Agricultural Development Co., Ltd. (national large-scale poultry food processing listed companies) made their products entered market without having their products in accordance with relevant provisions of harmless treatment in 2012.

\subsection{Summary}

From the statistical data, food comprehensive processing link is the highest frequency of food safety incidents, which should be highly valued. There are three reasons accounting for its occurrence.

1) In terms of food category, the high-price foods for their high profits, such as meat and meat products, liquor (especially high-priced wine and imported wine), aquatic products and other foods, exist serious cheating phenomena.

2) In the aspect of responsible subjects, the large and medium-sized enterprises do not match consumers' trust, in turn, the number of food safety incidents occurrence are basically the same with unlicensed operators. There are three possible reasons for it. Firstly, the probability that media cover large and medium-sized enterprises of food safety issues is higher than other-sized responsibility. Secondly, some large and medium-sized enterprises have management, systems and other loopholes, for their large scale, so some branches and staff may not be managed well. Thirdly, one of the possible reasons that the large and medium-sized enterprises occurs food safety events is that there are problems in business philosophy of managers.

3) From the influencing factors of food safety incidents perspective, the majority of large and medium-sized enterprises who add excessive and illegal use of additives in the production and processing is mainly caused by industry standards, laws and regulations are imperfect and exist loophole. While, individual businesses and unlicensed operators add excessive and illegal use of additives or other hazard for their insufficient knowledge of the relevant laws and regulations, weakly social responsibility as well as pursuing too much personal interests.

\section{Recommendations}

\subsection{Food Supply Link}

As the source of food supply chain, although there are a few food safety incidents occurring in food planting/breeding link, once problems happen in this link, then the links behind it can't continue. Therefore, we must vigorously develop green planting/breeding, and detect agricultural land and feed of farms rigorously. In addition, it's necessary to popularize and deepen knowledge, science and technology about food safety. At the same time, the government should implement strict inspection and quarantine system for the animal and plant into market to ensure no risk of each batch of raw materials into the market.

\subsection{Food Circulation Link}

There are mainly two problems in food circulation link. Firstly, in the process of transportation/storage, the improper control of temperature, humidity, light, packaging and other external conditions results in food spoilage. Secondly, the problem foods enter into market, which is caused by fault of food inspection and quarantine in food circulation. Therefore, it is recommended to increase the investment of equipment and technology in circulation area. At the same time, the relevant departments should strengthen the management of its staff, improve departmental rules and regulations, and take effective measures to quarantine misconduct in order to reduce the error rate.

\subsection{Food Processing Link}

As the core part of the food supply chain, food processing link is also hardest-hit area with high rate of incidence of food safety incidents. To reduce the risk of this link, businesses must work together with the relevant regula- 
tory authorities. On one hand, regulators should publish detailed regulations as externally imposed norms, and increase the inspection and quarantine in the process of production and processing. On the other hand, strict procurement, processing and detecting process regulations should be introduced and effectively implemented in internal enterprises. Now some meat processing companies implement the policy that inspection and quarantine officers are long-term presence in enterprises for the convenience of random inspection at any time, which should be adopted by all food processing companies. For unlicensed operators, the relevant departments must severely punish them and prosecute the flow of their products, to ensure that the problem food not flow into market.

\subsection{Food Consumption Link}

For food retail sectors, large and medium-sized supermarkets as well as individual businesses are the key supervision objects. On one hand, large and medium-sized supermarket, as the main place for public food procurement, should more strengthen self-discipline, while regulators should press supervision. On the other hand, individual businesses have scattered miscellaneous features, which makes difficult supervision, so to regulate the behavior of individual businesses, it's necessary to deepen their social responsibility and popularize related legal knowledge, at the same time, the government should raise the random inspection frequency and increase the punishment level.

In catering industry, there is much need to regulate. For instance, the raw material procurement should be guaranteed that there is no excessive pesticide residues, not expired metamorphism and not false; in the process of processing, there should be standard sanitation and without adding excessive food additives and other harmful substances; delivery process should have suitable storage conditions; the eaten food, waste oil and other used food of consumers are not sold to illegal traders and illicitly used secondary and so on. To ensure that the entire process of catering is safe and healthy, a series of detailed standards and regulations for each of the above links should be published to constrain every links of food chain.

\subsection{Food Recycling Link}

The problem foods in food recycling link are meat and meat products as well as edible oil, oil and its products. Therefore, it's advised that our government establishes a comprehensive recycling system of meat as well as edible oil, oil and its products and press effective implement, while the traders who illegally recycle and process food should be punished.

The food supply chain as a whole, only when every links are safe, can the entire food supply chain work smoothly and safely. In addition to above suggestions, from the whole food supply chain perspective, the government shall organize relevant departments to vigorously build complete food traceability systems to ensure that every problems in food supply chain have a basis to follow and subject to take responsibility for. Then the food supply chain will be transparent, consumers have more confidence in food consumption.

\section{Acknowledgements}

The paper are funded by National Social Science Fund Project of China "The Micro-mechanism of Formation, Prevention and Control for Food Supply Chain Risk Based on Behavioral Perspective" (NO. 13BGL062), and by Project supported by the Beijing Wuzi University canal scholars program "Prevention and Control for Food Supply Chain Risk" (No. 2013BWU1202-01).

\section{References}

[1] Gu, Y. and Shi, X. (2005) Regulatory Game of Food Supply Chain Link. China Food Drug Administration, 7, 5-8.

[2] Miao, R. (2013) A Research on Food Safety Regulatory Issues and Countermeasures in Circulation of China. China Business \& Trade, 9, 17-18.

[3] Sun, S. and Peng, Y. (2012) Study on the Coordination of Quality Safety Behavior in Breeding and Slaughtering Link within High Quality Pork Supply Chain. Issues in Agricultural Economy, 3, 77-83.

[4] Hastein, T., Hjeltnes, B. and Lillehaug, A., et al. (2006) Food Safety Hazards That Occur during the Production Stage: Challenges for Fish Farming and the Fishing Industry. Revue Scientifique et Technique (International Office of Epizootics), 25, 607-625. 
[5] Zhang, J., Li, Z. and Zou, J. (2013) The Key Points Analysis of Supply Chain Perspective of Food Quality and Safety Control. Logistics Engineering and Management, 10, 101-103.

[6] Li, H. (2012) Chinese Food Supply Chain Risk and Critical Control Points Analysis. Jiangsu Agricultural Sciences, 40, 262-264.

[7] Zhang, H., An, Y. and Zhang, W. (2013) A Research on Chinese Food Safety Risk Identification, Assessment and Management: Based on Empirical Analysis of Food Safety Incidents. Inquiry into Economic Issues, 6, 135-141.

[8] Liu, C., An, Y. and Zhang, W. (2013) Study on Weaknesses, Root Causes and Key Issues of China's Food Quality Safety: Based on the Empirical Analysis of 1460 Food Quality Safety Cases. Issues in Agricultural Economy, 6, 135141.

[9] Luo, L., An Y. and Gu, C., et al. (2013) Analysis of Sources of Risk and Regulatory Strategy of Chinese Food Safety. Journal of Food Science and Technology, 31, 77-82. 
Scientific Research Publishing (SCIRP) is one of the largest Open Access journal publishers. It is currently publishing more than 200 open access, online, peer-reviewed journals covering a wide range of academic disciplines. SCIRP serves the worldwide academic communities and contributes to the progress and application of science with its publication.

Other selected journals from SCIRP are listed as below. Submit your manuscript to us via either submit@scirp.org or Online Submission Portal.
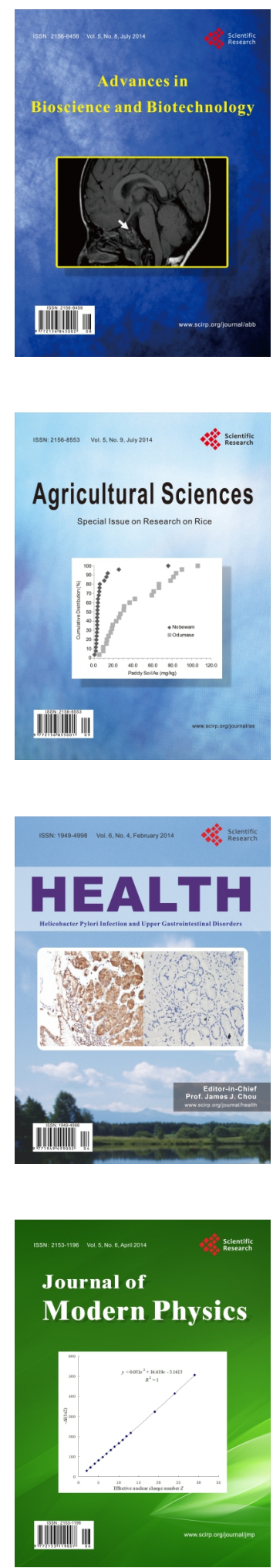
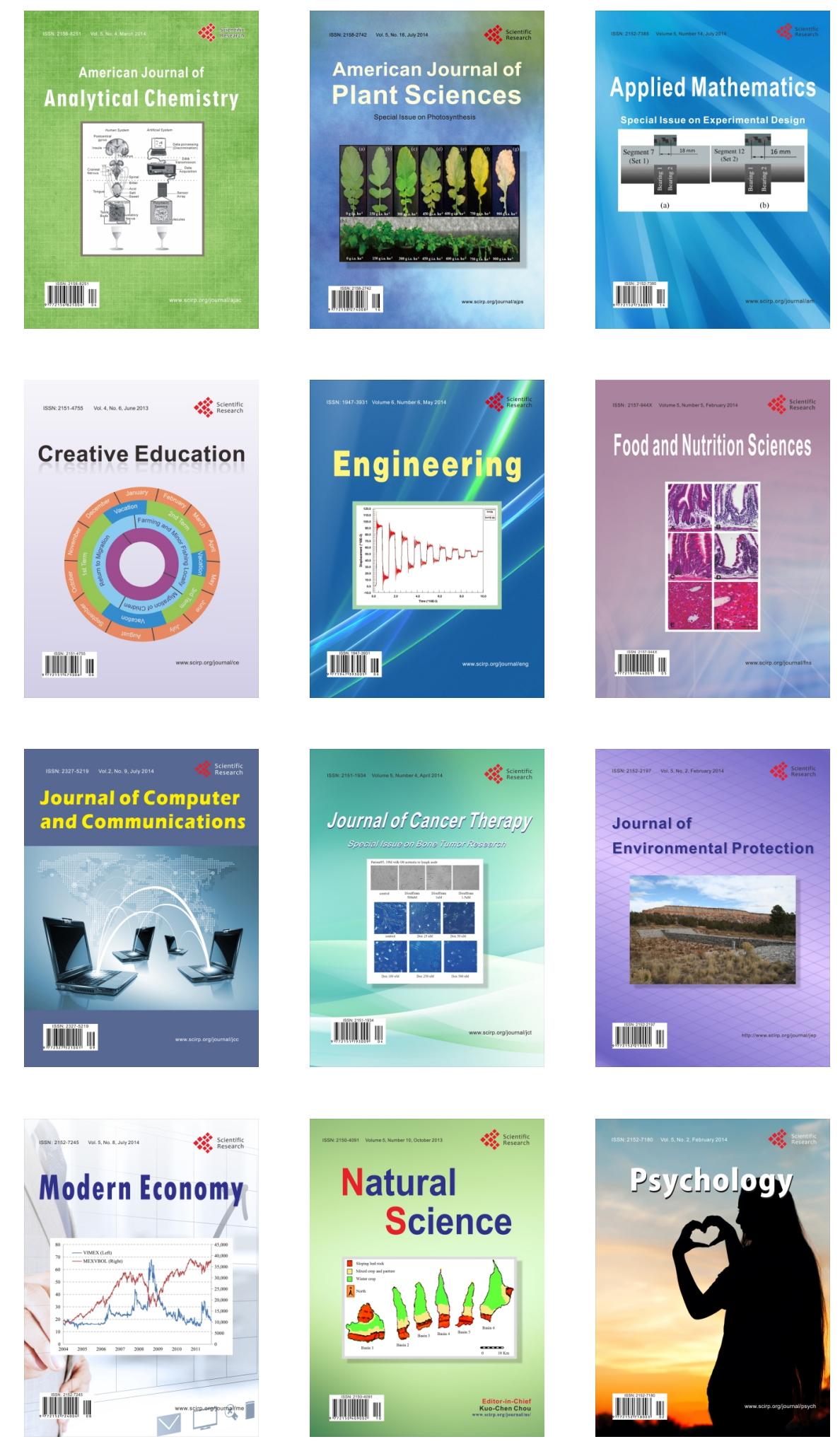